\title{
Collision rates for electron excitation of Mg V lines ${ }^{\star}$
}

\author{
S. S. Tayal and A. M. Sossah
}

\author{
Department of Physics, Clark Atlanta University, Atlanta, GA 30314, USA \\ e-mail: stayal@cau.edu
}

Received 9 October 2014 / Accepted 26 November 2014

\begin{abstract}
Aims. Transition probabilities and electron impact excitation collision strengths and rates for astrophysically important lines in Mg V are reported. The 86 fine-structure levels of the $2 s^{2} 2 p^{4}, 2 s 2 p^{5}, 2 p^{6}, 2 s^{2} 2 p^{3} 3 s, 2 s^{2} 2 p^{3} 3 p$ and $2 s^{2} 2 p^{3} 3 d$ configurations are included in our calculations. The effective collision strengths are presented as a function of electron temperature for solar and other astrophysical applications.

Methods. The collision strengths have been calculated using the B-spline Breit-Pauli R-matrix method for all fine-structure transitions among the 86 levels. The one-body mass, Darwin and spin-orbit relativistic effects are included in the Breit-Pauli Hamiltonian in the scattering calculations. The one-body and two-body relativistic operators are included in the multiconfiguration Hartree-Fock calculations of transition probabilities. Several sets of non-orthogonal spectroscopic and correlation radial orbitals are used to obtain accurate description of $\mathrm{Mg} \mathrm{V} 86$ levels and to represent the scattering functions.

Results. The calculated excitation energies are in very good agreement with experiment and represents an improvement over the previous calculations. The present collision strengths show good agreement with the previously available R-matrix and distorted-wave calculations. The oscillator strengths for E1 transitions normally compare very well with previous calculations. The thermally averaged collision strengths are obtained by integrating total resonant and non-resonant collision strengths over a Maxwellian distribution of electron energies and these are presented over the temperature range $\log _{10} T_{\mathrm{e}}=3.2-6.0 \mathrm{~K}$.
\end{abstract}

Key words. atomic data - atomic processes - line: formation

\section{Introduction}

Infrared and ultraviolet emission lines of $\mathrm{Mg}$ ions have been observed in the planetary nebula NGC 7207 (Russell et al. 1977; Grewing et al. 1978; Beckwith et al. 1984). The forbidden lines between the states of the $\mathrm{Mg} \mathrm{V}$ ground $2 \mathrm{~s}^{2} 2 \mathrm{p}^{4}$ configuration lie in the ultraviolet (UV) part of the spectrum and the lines due to the $2 s^{2} 2 p^{4}-2 s 2 p^{5}$ transitions appear in the extreme ultraviolet (EUV) spectrum of the solar corona, stellar atmospheres and other astrophysical plasmas. Electron impact excitation rates and transition probabilities are useful to determine electron temperatures and densities, ionization equilibria and for deriving elemental abundances from emission lines. The UV lines due to the forbidden $2 \mathrm{~s}^{2} 2 \mathrm{p}^{4}{ }^{3} \mathrm{P}_{2}-2 \mathrm{~s}^{2} 2 \mathrm{p}^{4}{ }^{1} \mathrm{D}_{2}$ and $2 \mathrm{~s}^{2} 2 \mathrm{p}^{4}{ }^{3} \mathrm{P}_{1}-2 \mathrm{~s}^{2} 2 \mathrm{p}^{4}$ ${ }^{1} S_{0}$ transitions at $2782 \AA$ and $1324 \AA$, respectively, were observed in the spectra of the Symbiotic star AG Draconis by the STIS spectrograph onboard the Hubble Space Telescope (Young et al. 2006). The intensity ratio of these Mg V UV lines is temperature and density sensitive and can be used for the diagnostic of astrophysical plasmas. The EUV line due to the allowed $2 \mathrm{~s}^{2} 2 \mathrm{p}^{4}{ }^{1} \mathrm{D}_{2}-2 \mathrm{~s} 2 \mathrm{p}^{5}{ }^{1} \mathrm{P}_{1}^{\mathrm{o}}$ transition occurs at $276.58 \AA$ and it has been observed by Hinode/EIS (Young et al. 2007; Brooks et al. 2009) and by SOHO/CDS. Several lines of O-like ions including $\mathrm{Mg} \mathrm{V}$ lines due to $2 \mathrm{~s}^{2} 2 \mathrm{p}^{4}-2 \mathrm{~s} 2 \mathrm{p}^{5}$ and $2 \mathrm{~s} 2 \mathrm{p}^{5}{ }^{1} \mathrm{P}^{\mathrm{o}}-2 \mathrm{p}^{6}{ }^{1} \mathrm{~S}$ transitions have been observed with the SERTS in the EUV spectrum of solar active regions (Thomas \& Neupert 1994). The

* Tables 1-4 are only available at the CDS via anonymous ftp to cdsarc.u-strasbg.fr $(130.79 .128 .5)$ or via

http://cdsarc.u-strasbg.fr/viz-bin/qcat?]/A+A/574/A87 intensity ratios between the forbidden $2 s^{2} 2 p^{4}{ }^{3} \mathrm{P}-2 \mathrm{~s}^{2} 2 \mathrm{p}^{4}{ }^{1} \mathrm{~S}$ transition and the high-excitation transitions $2 s^{2} 2 p^{3} 3 s-2 s^{2} 2 p^{3} 3 p$ and $2 s^{2} 2 p^{3} 3 p-2 s^{2} 2 p^{3} 3 d$ of O-like ions (Kink et al. 1997) are temperature sensitive and can be used to determine physical conditions in quiet and active regions of the solar corona.

Mendoza \& Zeippen (1987) reported electron excitation rates for transitions between the fine-structure $2 \mathrm{~s}^{2} 2 \mathrm{p}^{4}{ }^{3} \mathrm{P}_{0,1,2}$, ${ }^{1} \mathrm{D}_{2}$ and ${ }^{1} \mathrm{~S}_{0}$ levels from their close-coupling calculations. The LS coupled reactance matrices were transformed to intermediate coupling by ignoring the relativistic effects and by using the JAJOM program (Saraph 1972, 1978). Butler \& Zeippen (1994) performed a six-state $\left(2 \mathrm{~s}^{2} 2 \mathrm{p}^{4}{ }^{3} \mathrm{P},{ }^{1} \mathrm{D},{ }^{1} \mathrm{~S}, 2 \mathrm{~s} 2 \mathrm{p}^{5}{ }^{3} \mathrm{P}^{\mathrm{o}},{ }^{1} \mathrm{P}^{\mathrm{o}}\right.$ and $2 \mathrm{p}^{6}{ }^{1} \mathrm{~S}$ ) R-matrix calculation to obtain electron excitation collision strengths for O-like ions including $\mathrm{Mg} \mathrm{V}$. They also performed an algebraic transformation of LS reactance matrices to intermediate coupling. The energy levels and oscillator strengths of O-like ions have been measured using various experimental techniques. However, the electron excitation collision strengths have not yet been measured. The energy levels of $\mathrm{Mg} \mathrm{V}$ have been measured by Edlen (1964), Fawcett et al. (1974), Johannesson et al. (1972), Guennou et al. (1979) and Kaufman \& Artru (1980). The energy levels are compiled in NIST database ${ }^{1}$. The experimental oscillator strengths can be determined using measured lifetimes and branching ratios (Flaig \& Schartner 1985). Hudson et al. (2009) reported energies for the 19 LS states and 37 fine-structure levels and oscillator strengths for the allowed transitions between the 19 LS states in both length and velocity formulations using the CIV3 code (Hibbert 1975).

\footnotetext{
1 http://physics.nist.gov/physRefData/
} 
Tachiev \& Froese Fischer (2002) and Froese Fischer \& Tachiev (2004) calculated energy levels, transition rates and lifetimes using the multi-configuration Hartree-Fock (MCHF) method (Froese Fischer 2007). They included relativistic effects through the one-body and two-body Breit-Pauli operators.

Bhatia et al. (2006) reported energy levels, oscillator strengths, and collision strengths for $\mathrm{Mg} \mathrm{V}$. They performed a distorted-wave calculation and thus did not include resonances in their collision strengths. The effective collision strengths for transitions between fine-structure levels of the $2 s^{2} 2 p^{4}, 2 s 2 p^{5}$, $2 p^{6}, 2 s^{2} 2 p^{3} 3 s$ and $2 s^{2} 2 p^{3} 3 p$ configurations in $\mathrm{Mg} \mathrm{V}$ are reported by Hudson et al. (2009) from a 19-state (37 fine-structure levels) R-matrix calculation which may contain some uncertainties due to the omission of the levels of $2 s^{2} 2 p^{3} 3 d$ configuration of $n=3$ complex. The levels of $2 \mathrm{~s}^{2} 2 \mathrm{p}^{3} 3 \mathrm{~d}$ configuration exhibit strong mixing with lower levels. The better results can be obtained by improving the target description and by including additional levels of the $2 s^{2} 2 p^{3} 3 d$ configuration in the closecoupling expansion. New accurate calculations of electron excitation rates in the temperature range $10^{5}-10^{6} \mathrm{~K}$ for the UV and EUV lines are needed for applications to solar plasmas. The maximum $\mathrm{Mg} \mathrm{V}$ abundance in ionization equilibrium of solar plasmas occurs at round $T_{\mathrm{e}}=2.8 \times 10^{5} \mathrm{~K}$ (Bhatia et al. 2006). The purpose of our work is to provide highly accurate energy levels, oscillator strengths and electron excitation effective collision strengths for astrophysical applications. We included 86 levels of the $2 s^{2} 2 p^{4}, 2 s 2 p^{5}, 2 p^{6}, 2 s^{2} 2 p^{3} 3 s, 2 s^{2} 2 p^{3} 3 p$ and $2 s^{2} 2 p^{3} 3 d$ configurations of $\mathrm{Mg} \mathrm{V}$ in our MCHF calculations of transition rates and B-spline Breit-Pauli R-matrix calculations of electron excitation collision strengths. The target levels have been accurately represented by a set of spectroscopic and correlation non-orthogonal orbitals. The inclusion of additional resonance series converging to higher excited levels can change the effective collision strengths significantly for the $\mathrm{Mg} \mathrm{V}$ ion around the temperature of formation in solar plasmas.

\section{Excitation energies and oscillator strengths}

The terms of the $2 s^{2} 2 p^{4}, 2 s 2 p^{5}, 2 p^{6}, 2 s^{2} 2 p^{3} 3 s, 2 s^{2} 2 p^{3} 3 p$ and $2 s^{2} 2 p^{3} 3 d$ configurations of $\mathrm{Mg} \mathrm{V}$ were represented by the configuration-interaction (CI) wave functions using nonorthogonal orbitals in the MCHF approach (Froese Fischer 2007; Zatsarinny \& Froese Fischer 2000). The non-orthogonal orbitals were used to describe term dependence of valence orbitals as well as correlation and relaxation effects. The nonorthogonal orbitals provide flexibility in the choice of wave functions. Each target state wave function is represented by a linear combination of configuration state functions with the same $L S \pi$ symmetry as the target state. The target state wave functions for the $L S J$ levels are written as a sum over different $L S \pi$ value which couple to give the total $J \pi$ as follows,

$\Psi(\alpha J)=\sum_{j} a_{j} \Psi\left(\alpha_{j} L_{j} S_{j} J\right)$.

We obtained $1 \mathrm{~s}, 2 \mathrm{~s}$ and $2 \mathrm{p}$ orbitals for the ground $2 \mathrm{~s}^{2} 2 \mathrm{p}^{4}$ configuration terms in the Hartree-Fock (HF) calculations. We determined excited orbitals by optimization on different sates of even and odd parties using HF and MCHF methods. Separate sets of correlation orbitals $\overline{4 l}$ and $\overline{5 l}(l=0-4)$ were generated by optimization on the ground $2 \mathrm{~s}^{2} 2 \mathrm{p}^{4}{ }^{3} P$ and excited $2 \mathrm{~s} 2 \mathrm{p}^{5}{ }^{3} \mathrm{P}^{\mathrm{o}}$ states, respectively, to include all important correlation effects. The non-orthogonal orbitals were used in all main terms under consideration. In all other configurations of CI expansions a common set of orthogonal $1 \mathrm{~s}, 2 \mathrm{~s}, 2 \mathrm{p}, 3 \mathrm{~s}, 3 \mathrm{p}$ and $3 \mathrm{~d}$ orbitals was used. The CI expansions for different target states were produced using the spectroscopic and correlation orbitals in the above scheme by exciting up to three electrons to spectroscopic orbitals and up to two electrons to correlation orbitals from the basic configurations used in the description of target states. The CI expansions were reduced to a manageable size using a cut-off parameter of 0.004 for the expansion coefficients. The omission of insignificant configurations affected the relative excitation energies of the states by less than $0.1 \%$. A total of 5179 configurations were used to represent these states. We have included all important electron correlation effects for the target states under consideration. The CI expansions were further reduced for the representation of target states in the scattering calculations to 427 configurations by using a cut-off parameter of 0.02 . The absolute energy of the ground $2 s^{2} 2 p^{4}{ }^{3} \mathrm{P}_{2}$ level was -192.35138 au from the reduced CI expansion.

The present MCHF calculated excitation energies of the target states with 5179 configurations and with 427 configurations have been compared with the available measured values from the NIST compilation ${ }^{1}$ and other MCHF calculations of Tachiev $\&$ Froese Fischer ${ }^{2}$, Superstructure calculations of Bhatia et al. (2006) and Butler \& Zeippen (1994) and CIV3 calculations of Hudson et al. (2009). The present calculation of oscillator strengths included a total of 5179 configurations in CI expansions and one-body mass, Darwin and spin-orbit operators and two-body spin-other-orbit and spin-spin operators. We included mass correction, Darwin term and spin-orbit relativistic effects in the reduced 427 configurations calculation. The agreement between our two calculations is better than $1 \%$ for most states. Our MCHF calculations show excellent agreement with the measured values and with other MCHF calculations of Tachiev \& Froese Fischer ${ }^{2}$. The present calculation represents an improvement over the calculations of Bhatia et al. (2006), Hudson et al. (2009) and Butler \& Zeippen (1994). Hudson et al. (2009) reported lowest 37 fine-structure levels, while Butler \& Zeippen presented energies of the lowest ten levels. All other theoretical calculations used a set of the same orthogonal one-electron orbitals to represent all target states. We believe that our wave functions correctly represent the main correlation corrections, the interactions between different Rydberg series and term dependence effects.

The present oscillator strengths and transition probabilities for some E1 transitions from the states of ground $2 s^{2} 2 p^{4}$ configuration have been compared with the CI calculations of Tachiev \& Froese Fischer and Bhatia et al. in Table 2. The initial and final levels of a transition are given in Cols. 1 and 2 and in Cols. 3 and 4 we have listed the corresponding $(2 J+1)$ values of the initial and final levels. We have presented our length values of oscillator strengths and transition probabilities in Table 2. We have used experimental energies in the calculations of oscillator strengths and radiative rates. A rather reasonable agreement between different calculations is noted. Our MCHF calculation shows somewhat better agreement with the MCHF results of Tachiev \& Froese Fischer. We present oscillator strengths and transition probabilities for all E1 lines between the 86 fine-structure levels in Table 3 which is available on-line only. The relativistic effects in the structure calculations were included through the spin-orbit, spin-otherorbit, spin-spin, mass and Darwin Breit-Pauli operators in the Breit-Pauli Hamiltonian. We have presented oscillator strengths

http://nlte.nist.gov/MCHF/view.html 


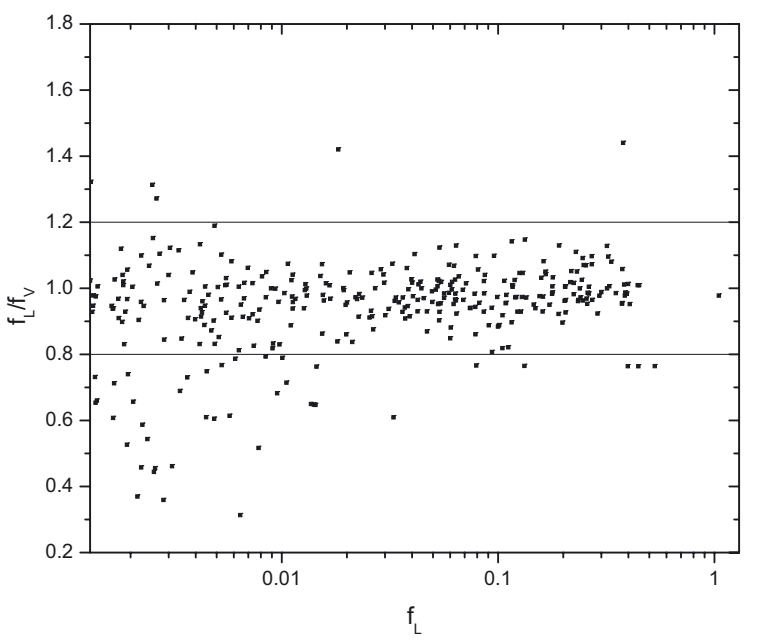

Fig. 1. Ratio between the present length and velocity oscillator strengths is shown as a function of length oscillator strengths. The horizontal lines indicate $20 \%$ deviation.

in both length and velocity formulations and transition probabilities in length form in Table 3. We have also listed the wavelength of each transition in this table. The values of oscillator strengths for intercombination lines are usually much smaller than those for the dipole-allowed transitions. The intercombination lines are produced by the spin-orbit interaction by causing mixing between different LS symmetries with the same set of quantum numbers $J$ and $\pi$. There is normally a very good agreement between the present length and velocity forms of oscillator strengths, particularly for strong transitions with significant strengths. The ratios of the present oscillator strengths in length and velocity formulations are displayed as a function of present length oscillator strengths in Fig. 1. The two horizontal lines show a deviation of $20 \%$. It is clear from the figure that the agreement between the present length and velocity values is within $20 \%$ or so for about $70 \%$ of the transitions. For some weaker transitions with oscillator strengths less than 0.05 the deviation between the length and velocity values is larger than $20 \%$. To some extent it is the indication of the good quality of our wave functions used in the calculations. The ratios of present length oscillator strengths with the results of Froese Fischer \& Tachiev (2004) have been shown in Fig. 2. The agreement between the two MCHF calculations is within $20 \%$ for many transitions. However, for a few transitions the two sets of results differ by about $50 \%$.

\section{Collision calculation}

We used B-spline basis for the description of continuum functions and did not impose any orthogonality constraint between continuum functions and the valence spectroscopic and correlated atomic orbitals (Zatsarinny \& Tayal 2002; Zatsarinny 2006). This allowed us to avoid potential inconsistencies between the continuum and bound parts of the close-coupling expansion. We included 86 fine-structure levels arising from the terms of the $2 \mathrm{~s}^{2} 2 \mathrm{p}^{4}, 2 \mathrm{~s} 2 \mathrm{p}^{5}, 2 \mathrm{p}^{6}$ and $2 \mathrm{~s}^{2} 2 \mathrm{p}^{3} 31(l=0-2)$ configurations in the close-coupling expansion. This allowed us to include channel coupling effects reasonably well between the levels of the $n=2$ and $n=3$ complexes. The theoretical approach and codes used in the calculation of collision strengths have been described by Zatsarinny (2006), and here we present a brief outline. The total wave function in the internal region is expanded

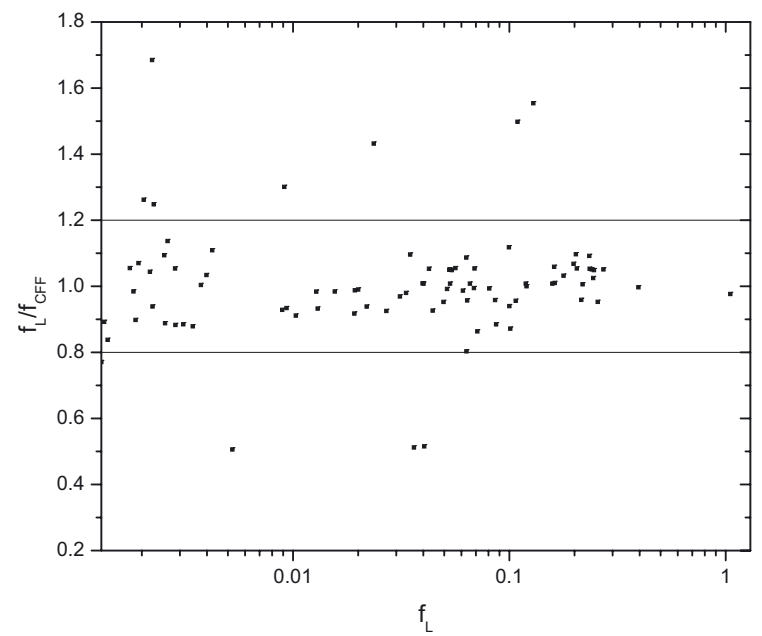

Fig. 2. Ratio between the present length oscillator strengths and results of Tachiev \& Froese Fischer is shown as a function of present length oscillator strengths. The horizontal lines indicate $20 \%$ deviation.

in terms of energy-independent functions

$$
\begin{aligned}
& \Psi_{k}\left(x_{1}, \ldots, x_{N+1}\right)= \\
& \mathcal{A} \sum_{i j} \bar{\Phi}_{i}\left(x_{1}, \ldots, x_{N} ; \hat{\mathbf{r}}_{N+1} \sigma_{N+1}\right) r_{N+1}^{-1} u_{j}\left(r_{N+1}\right) a_{i j k} \\
& \quad+\sum_{i} \chi_{i}\left(x_{1}, \ldots, x_{N+1}\right) b_{i k} .
\end{aligned}
$$

where $\overline{\Phi_{i}}$ are channel functions formed from the multiconfigurational functions of the 86 target levels and $u_{j}$ are the radial basis functions describing the motion of the scattering electron. The operator A antisymmetrizes the wave function and expansion coefficients $a_{i j k}$ and $b_{i k}$ are determined by diagonalizing the $(N+1)$-electron Hamiltonian. In our calculation, the radial functions $u_{j}$ are expanded in the $\mathrm{B}$-spline basis as

$u_{j}(r)=\sum_{i} \overline{a_{i j}} B_{i}(r)$,

and the coefficients $\overline{a_{i j}}$ (which now replace $a_{i j k}$ in Eq. (2)) are determined by diagonalizing the $(N+1)$-electron Hamiltonian inside the R-matrix box that contained all bound atomic orbitals used for the description of target states. The use of nonorthogonal orbitals allows us to reduce or even to avoid the introduction of additional $(N+1)$-electron terms in the R-matrix expansion. The latter may lead to extensive multi-configuration expansions, especially when correlated pseudo-orbitals are employed to improve the target states. This also allowed us to avoid the pseudo-resonance structure. The relativistic effects in the scattering calculations have been incorporated in the Breit-Pauli Hamiltonian through the use of the Darwin, mass correction and spin-orbit operators. The boundary radius of the R-matrix box was chosen to be $10.43 a_{0}\left(a_{0}=0.529 \times 10^{-10} \mathrm{~m}\right.$ and denotes the Bohr radius) and $61 \mathrm{~B}$-splines of order 8 were used for the expansion of continuum orbitals. These parameters provide converged results for an energy range up to $50.0 \mathrm{Ryd}$. The inner solutions at the boundary of the box were matched with asymptotic solutions in the outer region. The Seaton's STGF program as extended by Badnell (1999) has been employed to find the asymptotic solutions and collision strengths. The partial waves up to $2 J=59$ were calculated in the B-spline Breit-Pauli R-matrix calculations. A top-up procedure based on the Coulomb-Bethe 
approximation (Burgess \& Sheorey 1974) was employed to estimate the contributions for $2 J$ larger than 59 for the dipole allowed transitions. For the non-dipole transitions we have estimated the higher partial wave contributions by assuming that the collision strengths form a geometric progression in $J$.

The excitation rate coefficient for a transition from state $i$ to state $f$ at electron temperature $T_{\mathrm{e}}$ is given by

$C_{i f}=\frac{8.629 \times 10^{-6}}{g_{i} T_{\mathrm{e}}^{1 / 2}} \gamma_{i f}\left(T_{\mathrm{e}}\right) \exp \left(\frac{-\Delta E_{i f}}{k T_{\mathrm{e}}}\right) \mathrm{cm}^{3} \mathrm{~s}^{-1}$,

where $g_{i}$ is the statistical weight of the lower level $i, \Delta E_{i f}=$ $E_{f}-E_{i}$ is the excitation energy and the effective collision strength $\gamma_{i f}$ are calculated by integrating collision strengths $\Omega_{i f}$ for fine-structure levels over a Maxwellian distribution of electron energies

$\gamma_{i f}\left(T_{\mathrm{e}}\right)=\int_{0}^{\infty} \Omega_{i f} \exp \left(\frac{-E_{f}}{k T_{\mathrm{e}}}\right) \mathrm{d}\left(\frac{E_{f}}{k T_{\mathrm{e}}}\right)$,

where $E_{f}$ is the energy of incident electron with respect to the upper level $f$. The integration in Eq. (5) should be carried out using energy dependent collision strengths from threshold to infinity. The energy dependence of collision strengths for allowed transitions can be accounted by using numerical extrapolation technique. In the asymptotic region, the collision strengths follow a high energy limiting behavior for the dipole-allowed transitions

$\Omega_{i f}(E) \sim_{E \rightarrow \infty} \frac{4 S}{3} \ln (E)$,

where $S$ is the line strength. The collision strengths vary smoothly in the high energy region and exhibit an increasing trend for dipole-allowed transitions. For the electric quadrupole or magnetic-dipole non-dipole transitions the collision strengths remain fairly constant at higher energies.

\section{Collision strengths and effective collision strengths}

We have plotted collision strengths for the forbidden $2 s^{2} 2 \mathrm{p}^{4}{ }^{3} \mathrm{P}_{2}-$ $2 s^{2} 2 p^{4}{ }^{1} D_{2}(2782 \AA)$ and the dipole-allowed $2 s^{2} 2 p^{4}{ }^{1} D_{2}-2 s 2 p^{5}$ ${ }^{1} \mathrm{P}_{1}^{\mathrm{o}}(276 \AA)$ transitions of astrophysical importance as a function of incident electron energy in Figs. 3 and 4, respectively. The incident electron energy ranges from threshold to 50.0 Ryd and thus covers both resonant and non-resonant energy regions. The collision strengths display complex resonance structures in the closed-channels region at low incident incident electron energies. We used a fine-energy mesh of $5 \times 10^{-5}$ Ryd in the resonance energy region to resolve many narrow resonances. The collision strengths were calculated at a total of 34352 energy points. The resonance contributions are significantly larger and more complex for the forbidden transitions than for the allowed transitions. In the above-threshold energy region where all channels are opened, the collision strengths display smooth variation with electron energy for both forbidden and allowed transitions. Our results are compared with the distorted-wave calculation of Bhatia et al. (2006) who reported collision strengths at five electron energies of 10, 20, 30, 40 and 50 Ryd. All these energies lie in the above-threshold region where there are no resonances. The collision strengths for the forbidden $2 s^{2} 2 p^{4}{ }^{3} P_{2}-2 s^{2} 2 p^{4}{ }^{1} D_{2}$ transition (Fig. 3) slowly decrease with increasing electron energy in the above-threshold energy region and our calculation is in excellent agreement with the distorted-wave calculation. For the

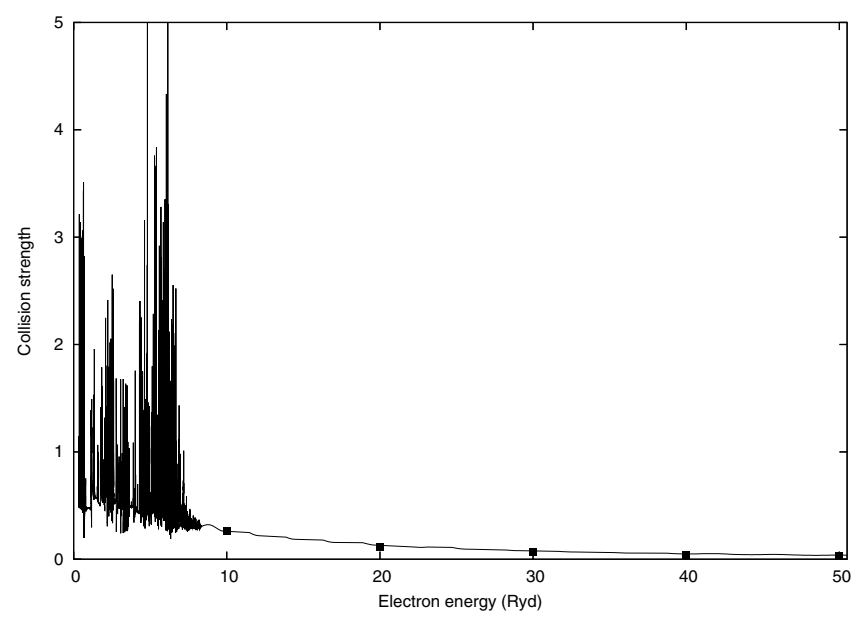

Fig. 3. Collision strengths for the forbidden $2 s^{2} 2 p^{4}{ }^{3} P_{2}-2 s^{2} 2 p^{4}{ }^{1} D_{2}$ (1-4) transition as a function of electron energy from threshold to 50 Ryd. Present results, solid curve; Bhatia et al. (2006), solid rectangles.

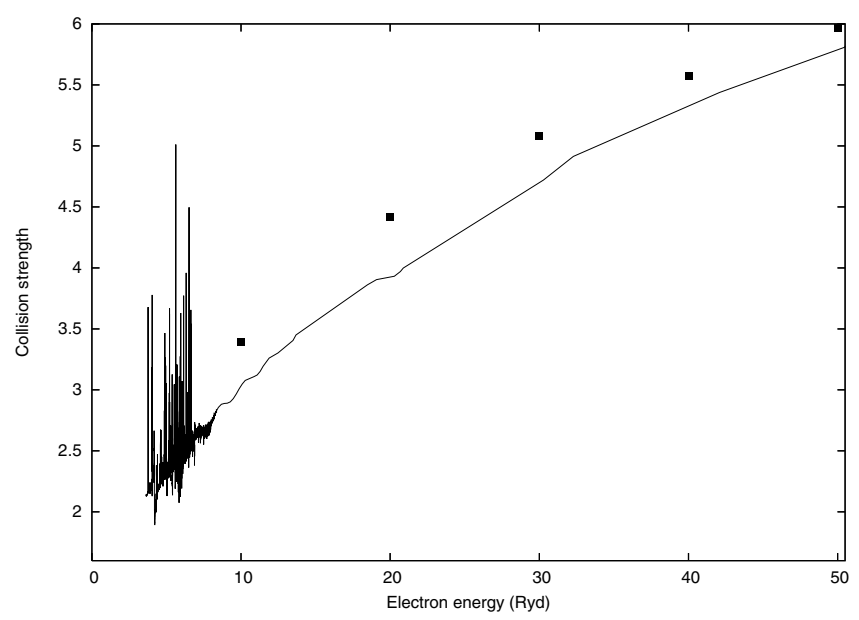

Fig. 4. Collision strengths for the allowed $2 \mathrm{~s}^{2} 2 \mathrm{p}^{4}{ }^{1} \mathrm{D}_{2}-2 \mathrm{~s} 2 \mathrm{p}^{5}{ }^{1} \mathrm{P}_{1}^{\mathrm{o}}$ (4-9) transition as a function of electron energy from threshold to 50 Ryd. Present results, solid curve; Bhatia et al. (2006), solid rectangles.

dipole-allowed $2 s^{2} 2 p^{4}{ }^{1} D_{2}-2 s 2 p^{5}{ }^{1} P_{1}^{o}$ transition (Fig. 4), the present collision strengths show qualitative agreement with the distorted-wave results but show some small quantitative differences. The collision strengths for the allowed transitions rise with increasing electron energy as expected. our results for the dipole-allowed $2 \mathrm{~s}^{2} 2 \mathrm{p}^{4}{ }^{1} \mathrm{D}_{2}-2 \mathrm{~s} 2 \mathrm{p}^{5}{ }^{1} \mathrm{P}_{1}^{\mathrm{o}}$ transition are lower by about $10 \%$ on an average from the results of Bhatia et al. (2006). It may be noted that the oscillator strengths from two calculations show similar differences with our oscillator strengths being smaller than those from Bhatia et al. (see Table 2).

We have plotted effective collision strengths for the forbidden $2 \mathrm{~s}^{2} 2 \mathrm{p}^{4}{ }^{3} \mathrm{P}_{2}-2 \mathrm{~s}^{2} 2 \mathrm{p}^{4}{ }^{3} P_{1}(1-2), 2 \mathrm{~s}^{2} 2 \mathrm{p}^{4}{ }^{3} \mathrm{P}_{2}-2 \mathrm{~s}^{2} 2 \mathrm{p}^{4}{ }^{1} \mathrm{D}_{2}$ (14), $2 \mathrm{~s}^{2} 2 \mathrm{p}^{4}{ }^{3} \mathrm{P}_{1}-2 \mathrm{~s}^{2} 2 \mathrm{p}^{4}{ }^{1} \mathrm{~S}_{0}(2-5)$ and $2 \mathrm{~s}^{2} 2 \mathrm{p}^{4}{ }^{1} \mathrm{D}_{2}-2 \mathrm{~s}^{2} 2 \mathrm{p}^{4}{ }^{1} \mathrm{~S}_{0}$ (4-5) transitions as a function of electron temperature in the range $\log _{10} T_{\mathrm{e}}=3.2-6.0 \mathrm{~K}$ in Figs. 5 and 6. Our results have been compared with the results from previous R-matrix calculations of Hudson et al. (2009) and Butler \& Zeippen (1994) and close-coupling calculation of Mendoza \& Zeippen (1984). A reasonable agreement between various calculations can be noted except for the $2 \mathrm{~s}^{2} 2 \mathrm{p}^{4}{ }^{3} \mathrm{P}_{1}-2 \mathrm{~s}^{2} 2 \mathrm{p}^{4}{ }^{1} \mathrm{~S}_{0}$ (2-5) transition where our results show significant differences with the calculation of Hudson et al. (2009). It may be noted that the effective 

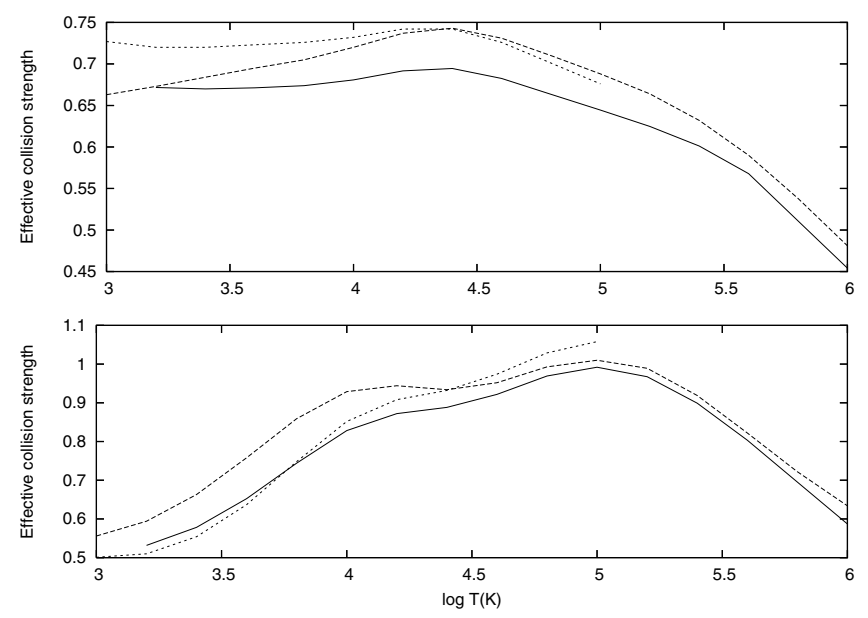

Fig. 5. Effective collision strengths for the forbidden $2 s^{2} 2 p^{4}{ }^{3} \mathrm{P}_{2}-2 \mathrm{~s}^{2} 2 \mathrm{p}^{4}$ ${ }^{3} \mathrm{P}_{1}(1-2)$ (lower panel) and $2 \mathrm{~s}^{2} 2 \mathrm{p}^{4}{ }^{3} \mathrm{P}_{2}-2 \mathrm{~s}^{2} 2 \mathrm{p}^{4}{ }^{1} \mathrm{D}_{2}$ (1-4) (upper panel) transitions as a function of electron temperature. Present results, solid curve; Hudson et al. (2009), long-dashed curve; Butler \& Zeippen (1994), short-dashed curve.
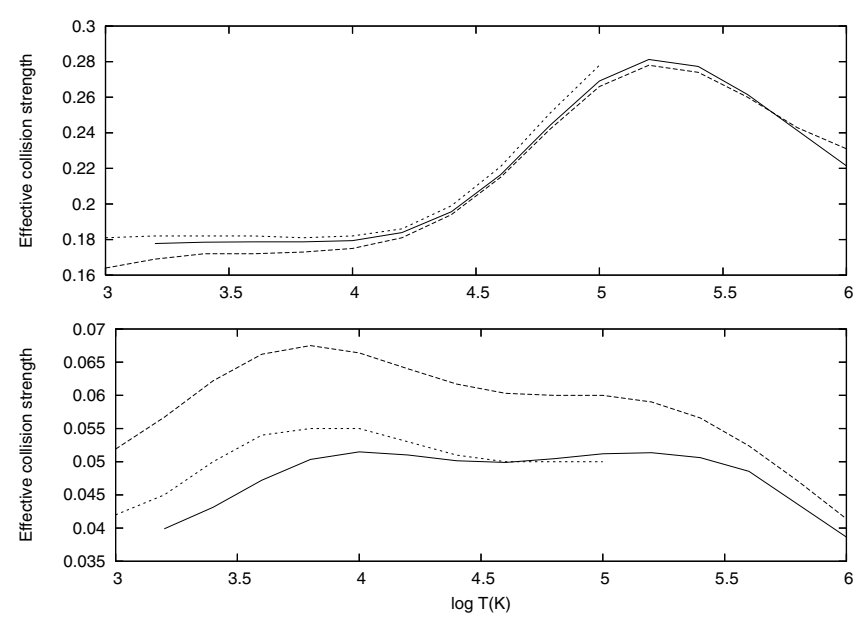

Fig. 6. Effective collision strengths for the forbidden $2 s^{2} 2 p^{4}{ }^{3} P_{1}-2 s^{2} 2 p^{4}$ ${ }^{1} \mathrm{~S}_{0}(2-5)$ (lower panel) and $2 \mathrm{~s}^{2} 2 \mathrm{p}^{4}{ }^{1} \mathrm{D}_{2}-2 \mathrm{~s}^{2} 2 \mathrm{p}^{4}{ }^{1} \mathrm{~S}_{0}$ (4-5) (upper panel) transitions as a function of electron temperature. Present results, solid curve; Hudson et al. (2009), long-dashed curve; Butler \& Zeippen (1994), short-dashed curve.

collision strength for this transition is almost an order smaller compared to other transitions shown here. The resonance structures in collision strengths make significant contributions to effective collision strengths at lower temperatures. The present effective collision strengths for the allowed $2 \mathrm{~s}^{2} 2 \mathrm{p}^{4}{ }^{3} \mathrm{P}_{2}-2 \mathrm{~s} 2 \mathrm{p}^{5}{ }^{3} \mathrm{P}_{1}^{\mathrm{o}}$ (1-7) and $2 s^{2} 2 p^{4}{ }^{1} D_{2}-2 s 2 p^{5}{ }^{1} P_{1}^{o}$ (4-9) transitions have been compared with previous R-matrix calculations of Hudson et al. (2009) in Fig. 7. The agreement between the two calculations is very good. We have presented effective collision strengths for all transitions between the lowest 86 fine-structure levels considered in our work in Table 4. The indices of lower and upper levels of a transition are given in Table 1 . The results are presented at 14 electron temperatures in the range $\log _{10} T_{\mathrm{e}}=$ 3.2-6.0 K suitable for modeling of stellar and solar corona and nebular plasmas. The Table 4 is available on-line only.
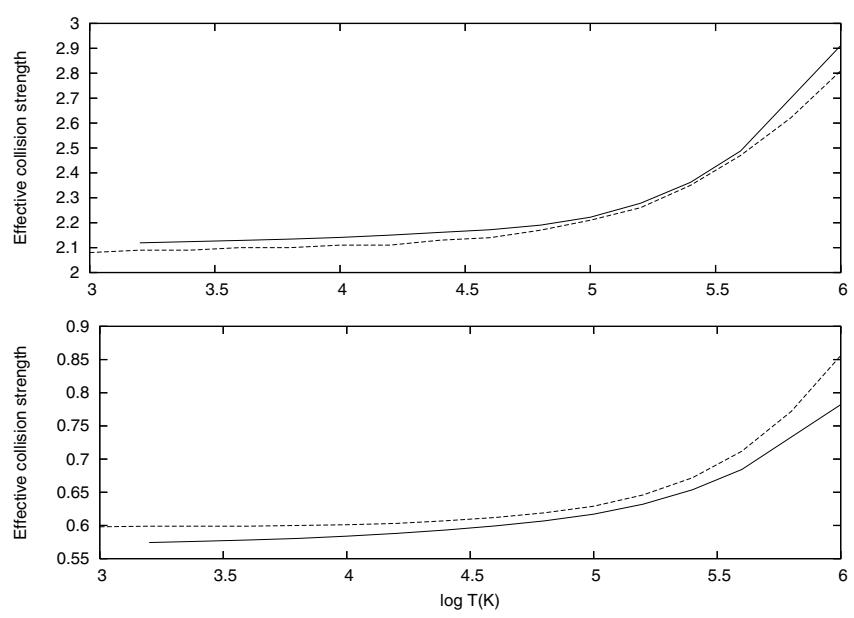

Fig. 7. Effective collision strengths for the allowed $2 \mathrm{~s}^{2} 2 \mathrm{p}^{4}{ }^{3} \mathrm{P}_{2}-2 \mathrm{~s} 2 \mathrm{p}^{5}$ ${ }^{3} \mathrm{P}_{1}^{\mathrm{o}}(1-7)$ (lower panel) and $2 \mathrm{~s}^{2} 2 \mathrm{p}^{4}{ }^{1} \mathrm{D}_{2}-2 \mathrm{~s} 2 \mathrm{p}^{5}{ }^{1} \mathrm{P}_{1}^{\mathrm{o}}$ (4-9) (upper panel) transitions as a function of electron temperature. Present results, solid curve; Hudson et al. (2009), dashed curve.

\section{Discussion and conclusions}

The target description and the convergence of close-coupling expansion are generally two major sources of error in any closecoupling scattering calculation. A comparison of calculated excitation energies and oscillator strengths with experiments and other reliable calculations as well as the fact that we used a well tested approach, our target description should be of high quality. A sufficient number of target states in the close-coupling expansion are required to achieve convergence for the transitions of interest. The collision strengths for transitions between the lower levels of the $2 s^{2} 2 p^{4}, 2 s 2 p^{5}, 2 p^{6}, 2 s^{2} 2 p^{3} 3 s$ and $2 s^{2} 2 p^{3} 3 p$ configurations are well converged in our calculation. The transitions from the level of the ground $2 s^{2} 2 p^{4}$ configuration to the levels of the $2 s^{2} 2 p^{4}, 2 s 2 p^{5}, 2 p^{6}, 2 s^{2} 2 p^{3} 3 s$ and $2 s^{2} 2 p^{3} 3 p$ configurations should be most accurate (10\% or better). The results are somewhat less accurate $(\sim 20 \%)$ for transitions between excited levels of the above configurations. For higher levels of the $2 s^{2} 2 p^{3} 3 d$ configuration there may be significant coupling effects from higher states and thus our results for transitions involving $2 s^{2} 2 p^{3} 3 d$ levels may be less accurate ( $25 \%$ or worse).

In conclusion, we have presented accurate oscillator strengths and effective collision strengths for $\mathrm{Mg} \mathrm{V}$ lines among the 86 fine-structure levels. We used non-orthogonal orbitals for the construction of CI wave functions for target levels and for the representation of scattering functions. Our target wave functions are likely to be accurate to yield reliable collision strengths from the B-spline Breit-Pauli R-matrix scattering calculations. We have attempted to account for important physical effects such as electron correlation, relativistic, and channel coupling effects. The complex resonance series converging to several excited levels make significant contributions to collision strengths. The effective collision strengths are presented over a wide range of electron temperatures suitable for modeling of emissions from various types of astrophysical plasmas.

Acknowledgements. This research work is supported by NASA grant NNX11AB62G from the Solar and Heliophysics program.

\section{References}

Badnell, N. R. 1999, J. Phys. B, 32, 5583

Beckwith, S., Evans, N. J., Natta, A., Russell, R. W., \& Wyant, J. 1984, ApJ, 277,207 
Bhatia, A. K., Landi, E., \& Eissner, W. 2006, At. Data Nucl. Data Tables, 92, 105

Brooks, D. H., Warren, H. P., Williams, R., \& Watanabe, T. 2009, ApJ, 705, 1522 Burgess, A., \& Sheorey, V. B. 1974, J. Phys. B, 7, 2403

Butler, K., \& Zeippen, C. J. 1994, A\&AS, 108, 1

Edlen, B. 1964, in Encyclopedia of Physics (Heidelberg: Springer), ed. S. Flugge, 27, 172

Eissner, W., Jones, M., \& Nussbaumer, H. 1974, Comput. Phys. Commun., 8, 270

Fawcett, B. C., Galanti, M., \& Peacock, J. 1974, J. Phys. B, 7, 1149

Flaig, H. J., \& Schartner, K. H. 1985, Phys. Scr., 31, 255

Froese Fischer, C. 2007, Comp. Phys. Commun., 176, 559

Froese Fischer, C., \& Tachiev, G. I. 2004, At. Data Nucl. Data Tables, 87, 1

Grewing, M., Boksenberg, A., Seaton, M. J., et al. 1978, Nature, 275, 394

Guennou, H., Surreau, A., Carillon, A., \& Jamelot, G. 1979, J. Phys. B, 12, 1657

Hibbert, A. 1975, Comput. Phys. Commun., 9, 141
Hudson, C. E., Ramsbottom, C. A., Norrington, P. H., \& Scott, M. P. 2009, A\&A, 494, 729

Johannesson, G. A., Lundstrom, T., \& Minnhagen, L. 1972, Phys. Scr., 6, 129

Kaufman, V., \& Artru, M.-C. 1980, J. Opt. Soc. Am., 70, 1135

Kink, I., \& Engström, L. 1997, Phys. Scr., 56, 31

Mendoza, C., \& Zeippen, C. J. 1987, MNRAS, 224, 7

Russell, R. W., Soifer, B. T., \& Willner, S. P. 1977, ApJ, 217, L149

Saraph, H. E. 1972, Comput. Phys. Commun., 3, 256

Saraph, H. E. 1978, Comput. Phys. Commun., 15, 247

Tachiev, G. I., \& Froese Fischer, C. 2002, A\&A, 385, 716

Thomas, R. J., \& Neupert, W. M. 1994, ApJS, 91, 461

Young, P. R., Dupree, A. K., Espey, B. R., \& Kenyon, S. J. 2006, ApJ, 650, 1091

Young, P. R., Del Zanna, G., Mason, H. E., et al. 2007, PASJ, 59, 727

Zatsarinny, O. 2006, Comput. Phys. Commun., 174, 273

Zatsarinny, O., \& Froese Fischer, C. 2000, Comput. Phys. Commun., 123, 247

Zatsarinny, O., \& Tayal, S. S. 2002, J. Phys. B, 35, 241 